\title{
STUDY OF MULTIPLE STRUCTURES ON PROJECTIVE SUBVARIETIES
}

\author{
M. R. GONZALEZ-DORREGO \\ Departamento de Matemáticas, Universidad Autónoma de Madrid \\ 28049 Madrid, Spain \\ ORCID:0000-0002-2706-7024Ｅ-mail: mrosario.gonzalez@uam.es
}

\begin{abstract}
Let $k$ an algebraically closed field, char $k=0$.
We study multiplicity- $r$ structures on varieties for $r \in \mathbb{N}, r \geq 2$. Let $Z$ be a reduced irreducible nonsingular $(N-2)$-dimensional variety such that $r Z=X \cap F$, where $X$ is a normal $(N-1)$ fold of degree $n, F$ is a smooth $(N-1)$-fold of degree $m$ in $\mathbb{P}^{N}$, such that $r \in \mathbb{N}, r \geq 2$, $Z \cap \operatorname{Sing}(X) \neq \emptyset$. There are effective divisors $V$ and $D_{1}$ on $Z$ such that $O_{Z}\left(V-(r-1) D_{1}\right) \simeq$ $\omega_{Z}{ }^{r}(-r m-n+(N+1) r)$, where $\omega_{Z}$ is the canonical sheaf of $Z$.
\end{abstract}

Let $Z \subset \mathbb{P}^{N}$ be a reduced irreducible subvariety of codimension 2 . Let $Y$ be an irreducible hypersurface in $\mathbb{P}^{N}, Z \subset Y$. Let $\omega^{o}{ }_{Z}$ be the dualizing sheaf of $Z$. Then, there exists a hypersurface $X$ in $\mathbb{P}^{N}$ such that $Z=Y \cap X$ is a scheme-theoretical complete intersection if and only if

- $\left.\omega^{o}{ }_{Z} \simeq \omega_{\mathbb{P}^{N}} \otimes \wedge^{2} \mathcal{N}_{Z}\right|_{\mathbb{P}^{N}}$.

- $\operatorname{deg} Y$ divides $\operatorname{deg} Z$.

- $\omega^{o}{ }_{Z} \simeq O_{Z}\left(\operatorname{deg} Y+\left(\frac{\operatorname{deg} Z}{\operatorname{deg} Y}\right)-N-1\right)$.

Introduction. There are two sections in this paper. In Section 1 we study multiplicity- $r$ structures on varieties $r \in \mathbb{N}$.

Let $k$ be an algebraically closed field of characteristic 0. Most of our results could also be extended to char $k=p, p>0$. Let $Y$ be a nonsingular variety in $\mathbb{P}^{N}$, with ideal sheaf $I_{Y}$. A non-reduced structure $\tilde{Y}$ is a multiplicity-r structure on $Y$ if

- $I_{\tilde{Y}} \subset I_{Y}$;

- $\tilde{Y}$ is locally a complete intersection;

2010 Mathematics Subject Classification: 14B05, 14E15, 32S25, 14J17, 14J30, 14J35, 14J40.

Key words and phrases: multiplicity structures, dualizing sheaf, complete intersection.

The paper is in final form and no version of it will be published elsewhere. 
- $\tilde{Y}$ has multiplicity $r$, i.e. for each point $P \in Y$ and a general hyperplane $H$ through $P$, the local intersection multiplicity is

$$
i(P ; \tilde{Y}, H)=\operatorname{dim} \frac{O_{P}}{I(\tilde{Y} \cap H)}=r .
$$

Multiplicity-2 structures on nonsingular varieties appear in several instances; for example, when studying nonsingular curves on a Kummer surface in $\mathbb{P}^{3}$, passing through some of its nodes. To define a multiplicity- 2 structure $\tilde{Y}$ on a codimension 2 nonsingular variety $Y$ is, under some conditions, equivalent to defining a subbundle $L \subset N_{Y \mid \mathbb{P}^{n}}$, assuming that $I_{Y} / I_{\tilde{Y}}$ is locally free. W. Barth gave a construction of the Horrocks-Mumford bundle assuming the existence of a nonsingular irreducible curve with certain properties; among them a double structure on the curve (see [2]). The Horrocks-Mumford bundle is a stable indecomposable rank 2 vector bundle over $\mathbb{P}^{4}$. The study of varieties which are complete intersections with a non-reduced structure on them could be used in the construction of vector bundles in $\mathbb{P}^{n}$.

Multiple structures on curves ('thickenings of curves') appear in the enumerative geometry of Calabi-Yau $n$-folds. In particular, Gromov-Witten theory can be used to define an enumerative geometry of curves in Calabi-Yau 5-folds (see [6]). Also thickenings of rational curves, analytically contractible, on Calabi-Yau 3-folds are used to study the genus zero Gopakumar-Vafa invariants (see [5]).

Let $Z$ be a reduced irreducible nonsingular $(N-2)$-dimensional variety such that $r Z=X \cap F, r \in \mathbb{N}, r \geq 2$, where $F$ is a $(N-1)$-fold in $\mathbb{P}^{N}, X$ is a normal $(N-1)$-fold with $Z \cap \operatorname{Sing}(X) \neq \emptyset$. We prove that there are effective divisors $V$ and $D_{1}$ on $Z$ such that $O_{Z}\left(V-(r-1) D_{1}\right) \simeq \omega_{Z}{ }^{r}(-r m-n+(N+1) r)$, where $\omega_{Z}$ is the canonical sheaf of $Z$.

In Section 2 we give conditions for a reduced irreducible subvariety $Z$, in $\mathbb{P}^{N}$, of codimension 2, to be a scheme-theoretical complete intersection. Our motivation is the extension of a type of Noether-Lefschetz Theorem from surfaces to $n$-folds (see [4]).

We would like to thank the Department of Mathematics at the University of Toronto for their hospitality during the preparation of this manuscript and Mark Spivakovsky for useful discussions. We also thank the referee for valuable comments.

1. Let $Z$ be a reduced irreducible nonsingular $(N-2)$-dimensional variety such that $r Z=X \cap F$, where $X$ is a normal $(N-1)$-fold, $F$ is a smooth $(N-1)$-fold in $\mathbb{P}^{N}$, such that $r \in \mathbb{N}, r \geq 2, Z \cap \operatorname{Sing}(X) \neq \emptyset$. Let $m$ be the degree of $F$ and $n$ be the degree of $X$. Let $I_{Z}$ be the ideal sheaf of $Z$ in $\mathbb{P}^{N}$.

Let us write $T=r Z, r \in \mathbb{N}, r \geq 2$. Consider the Cohen-Macaulay filtration

$$
Z=T_{1} \subset T_{2} \subset \ldots \subset T_{r}=r Z, \quad \text { where } T_{i}=i Z, 1 \leq i \leq r .
$$

Let $J_{i}=I_{Z}{ }^{i}+O_{\mathbb{P}^{N}}(-F), 1 \leq i \leq r$. Note that $I_{Z}=J_{1}, I_{Z}^{2} \subset J_{2} \subset I_{Z} \cdot \frac{J_{i}}{J_{(i+1)}}$ is a rank 1 locally free $O_{Z}$-module. $\frac{J_{i}}{I_{Z} J_{i}}$ is a rank 2 locally free $O_{Z}$-module, $1 \leq i \leq r$.

We generalize some results of [1]. 
Let $\mathcal{L}=\frac{I_{Z}}{J_{2}}$. We consider the exact sequence

$$
0 \rightarrow \frac{J_{2}}{I_{Z}^{2}} \rightarrow \frac{I_{Z}}{I_{Z}^{2}} \rightarrow \mathcal{L} \rightarrow 0
$$

$\mathcal{L}$ is a quotient of $\frac{I_{Z}}{I_{Z}{ }^{2}}$, the conormal bundle of $Z$ in $\mathbb{P}^{N} \cdot \frac{I_{Z}}{I_{Z}{ }^{2}}$ is a locally free sheaf of rank 2 over $O_{Z}=O_{\mathbb{P}^{N}} / I_{Z}$.

Since $Z$ is contained in the hypersurface $F$ of degree $m$, for $I_{F}=O_{\mathbb{P}^{N}}(-m)$, we have

$$
0 \rightarrow O_{\mathbb{P}^{N}}(-m) \rightarrow I_{Z}
$$

which, by restriction to $Z$, gives $0 \rightarrow O_{Z}(-m) \rightarrow \frac{I_{Z}}{I_{Z}{ }^{2}}$, since $\operatorname{Tor}_{1}\left(\frac{I_{Z}}{O_{\mathbb{P}^{N}}(-m)}, O_{Z}\right)=0$.

We also have

$$
0 \rightarrow O_{\mathbb{P}^{N}}(-m) \rightarrow J_{2} \rightarrow I_{Z}
$$

Restricting (2) to $Z$, we have $O_{Z}(-m) \rightarrow \frac{J_{2}}{I_{Z} J_{2}} \rightarrow \frac{I_{Z}}{I_{Z}^{2}}$.

Let $M_{1}=: \frac{J_{2}}{I_{Z}{ }^{2}}$. It is a rank 1 locally free sheaf on $Z$ since so it is $\mathcal{L}$ and $(1)$ is exact. Let $\alpha: O_{Z}(-m) \rightarrow \frac{I_{Z}}{I_{Z}{ }^{2}}$. Let $\delta: \frac{I_{Z}}{I_{Z}{ }^{2}} \rightarrow$ Coker $\alpha$.

We also have

$$
\begin{gathered}
0 \rightarrow O_{Z}(-m) \rightarrow \frac{J_{2}}{I_{Z}^{2}} \rightarrow \frac{M_{1}}{O_{Z}(-m)} \rightarrow 0, \\
0 \rightarrow \frac{M_{1}}{O_{Z}(-m)} \rightarrow \text { Coker } \alpha \rightarrow \mathcal{L} \rightarrow 0 .
\end{gathered}
$$

Let $\gamma:$ Coker $\alpha \rightarrow \mathcal{L} \rightarrow 0$. Let us consider $\gamma \circ \delta$. Hence, there exists an effective divisor $D_{1}$ on $Z$ such that

$$
0 \rightarrow O_{Z}\left(-m+D_{1}\right) \rightarrow \frac{I_{Z}}{I_{Z}^{2}} \rightarrow \mathcal{L} \rightarrow 0
$$

Thus, $M_{1}=O_{Z}\left(D_{1}-m\right)$.

Taking exterior powers in the exact sequence

$$
\left.0 \rightarrow \frac{I_{Z}}{I_{Z}^{2}} \rightarrow \Omega\right|_{\mathbb{P}^{N}} \otimes O_{Z} \rightarrow \omega_{Z} \rightarrow 0
$$

we obtain $\wedge^{2}\left(\frac{I_{Z}}{I_{Z}}\right) \simeq \omega_{Z}{ }^{-1}(-(N+1))$.

From the latter and the exact sequence (1),

$$
\mathcal{L}=\frac{I_{Z}}{J_{2}} \simeq \wedge^{2}\left(\frac{I_{Z}}{I_{Z}^{2}}\right) \otimes M_{1}^{\otimes-1} \simeq \omega_{Z}{ }^{-1}(-(N+1)) \otimes O_{Z}\left(m-D_{1}\right) .
$$

We can obtain similar result from (3).

Consider the exact sequence

$$
\begin{gathered}
0 \rightarrow \frac{I_{Z}^{2}}{I_{Z} J_{2}} \rightarrow \frac{J_{2}}{I_{Z} J_{2}} \rightarrow \frac{J_{2}}{I_{Z}^{2}} \rightarrow 0 . \\
\wedge^{2}\left(\frac{J_{2}}{I_{Z} J_{2}}\right) \simeq \frac{I_{Z}^{2}}{I_{Z} J_{2}} \otimes \frac{J_{2}}{I_{Z}^{2}} \simeq \omega_{Z}^{-2}(-2(N+1)) \otimes O_{Z}\left(m-D_{1}\right),
\end{gathered}
$$

since $\left(\frac{I_{Z}}{J_{2}}\right)^{\otimes 2} \simeq \frac{I_{Z}^{2}}{I_{Z} J_{2}}$ and $\left(\frac{I_{Z}}{J_{2}}\right)^{\otimes 2} \simeq \omega_{Z}{ }^{-2}(-2(N+1)) \otimes O_{Z}\left(2 m-2 D_{1}\right)$.

We also have

$$
0 \rightarrow O_{\mathbb{P}^{N}}(-m) \rightarrow J_{3} \rightarrow J_{2} \rightarrow I_{Z}
$$


After restricting to $Z$, we have $O_{Z}(-m) \rightarrow \frac{J_{3}}{I_{Z} J_{3}} \rightarrow \frac{J_{2}}{I_{Z} J_{2}}$. Let $\mu_{1}$ be their composition; $\mu_{1}: O_{Z}(-m) \rightarrow \frac{J_{2}}{I_{Z} J_{2}}$. Consider $\mu_{11}: \frac{J_{2}}{I_{Z} J_{2}} \rightarrow$ Coker $\mu_{1}$.

Consider the exact sequence

$$
0 \rightarrow \frac{J_{3}}{I_{Z} J_{2}} \rightarrow \frac{J_{2}}{I_{Z} J_{2}} \rightarrow \frac{J_{2}}{J_{3}} \rightarrow 0 .
$$

Then $M_{2} \simeq \frac{J_{3}}{I_{Z} J_{2}}$ is a rank 1 locally free sheaf on $Z$. Let $\mu_{12}: O_{Z}(-m) \rightarrow \frac{J_{3}}{I_{Z} J_{2}}$. The image of $\mu_{1}$ maps to zero in $\frac{J_{2}}{J_{3}}$. We have an exact sequence

$$
0 \rightarrow \text { Coker } \mu_{12} \rightarrow \text { Coker } \mu_{1} \rightarrow \frac{J_{2}}{J_{3}} \rightarrow 0 .
$$

Then there exists an effective divisor $D_{2}$ such that $M_{2}=O_{Z}\left(D_{2}-m\right)$ since

$$
0 \rightarrow O_{Z}(-m) \rightarrow M_{2} \rightarrow \frac{M_{2}}{O_{Z}(-m)} \rightarrow 0,
$$

and

$$
0 \rightarrow O_{Z}\left(-m+D_{2}\right) \rightarrow \frac{J_{2}}{I_{Z} J_{2}} \rightarrow \frac{J_{2}}{J_{3}} \rightarrow 0
$$

$D_{2}$ is the divisor associated to the torsion subsheaf $\frac{M_{2}}{O_{Z}(-m)}$.

We have

$$
\begin{gathered}
\frac{J_{2}}{J_{3}} \simeq \wedge^{2}\left(\frac{J_{2}}{I_{Z} J_{2}}\right) \otimes M_{2}{ }^{\otimes-1}, \\
\frac{J_{2}}{J_{3}} \simeq \omega_{Z}{ }^{-2}(-2(N+1)) \otimes O_{Z}\left(2 m-D_{1}-D_{2}\right) .
\end{gathered}
$$

Notice that $\left(\frac{I_{Z}}{J_{2}}\right)^{\otimes 2} \simeq \frac{I_{Z}^{2}}{I_{Z} J_{2}}$. We have

$$
0 \rightarrow \frac{I_{Z}^{2}}{I_{Z} J_{2}} \rightarrow \frac{J_{2}}{I_{Z} J_{2}}
$$

Consider the exact sequence (5). There exists an effective divisor $N_{1}$ on $Z$ such that

$$
\frac{J_{2}}{J_{3}} \simeq\left(\frac{I_{Z}}{J_{2}}\right)^{\otimes 2}\left(N_{1}\right) \text {. }
$$

Let $M_{r-1} \simeq \frac{J_{r}}{I_{Z} J_{(r-1)}}$ which is a rank 1 locally free sheaf on $Z$. As in (4), we also have

$$
0 \rightarrow O_{\mathbb{P}^{N}}(-m) \rightarrow J_{r} \rightarrow J_{(r-1)} \rightarrow I_{Z}
$$

After restricting to $Z$ the map $0 \rightarrow O_{\mathbb{P}^{N}}(-m) \rightarrow J_{(r-1)}$, we have $\mu_{(r-2)}: O_{Z}(-m) \rightarrow$ $\frac{J_{(r-1)}}{I_{Z} J_{(r-1)}}$.

Consider $\mu_{(r-2) 1}: \frac{J_{(r-1)}}{I_{Z} J_{(r-1)}} \rightarrow$ Coker $\mu_{(r-2)}$. Let $\mu_{(r-2) 2}: O_{Z}(-m) \rightarrow \frac{J_{r}}{I_{Z} J_{(r-1)}}$. The image of $\mu_{(r-2)}$ maps to zero in $\frac{J_{(r-1)}}{J_{r}}$.

We have an exact sequence $0 \rightarrow$ Coker $\mu_{(r-2) 2} \rightarrow$ Coker $\mu_{(r-2)} \rightarrow \frac{J_{(r-1)}}{J_{r}} \rightarrow 0$. We deduce that there exists an effective divisor $D_{(r-1)}$ such that $M_{(r-1)}=O_{Z}\left(D_{(r-1)}-m\right)$. Proposition 1. $\frac{J_{(r-1)}}{J_{r}} \simeq\left(\frac{I_{Z}}{J_{2}}\right)^{\otimes(r-1)}\left(N_{(r-2)}\right), r \geq 3$, where $N_{(r-2)}$ is an effective divisor on $Z$. Also, $\wedge^{2}\left(\frac{J_{(r-1)}}{I_{Z} J_{(r-1)}}\right) \simeq \omega_{Z}{ }^{-(r-1)}(-(N+1)(r-1)) \otimes O_{Z}\left((r-2) m-(r-1) D_{1}+D_{(r-1)}\right)$, where $D_{1}$ and $D_{(r-1)}$ are effective divisors on $Z$. 
Proof. For $N=3$, see 1 .

Let $N \geq 3$. We have an exact sequence

$$
0 \rightarrow \frac{J_{r}}{I_{Z} J_{(r-1)}} \rightarrow \frac{J_{(r-1)}}{I_{Z} J_{(r-1)}} \rightarrow \frac{J_{(r-1)}}{J_{r}} \rightarrow 0
$$

Notice that $\left(\frac{I_{Z}}{J_{2}}\right)^{\otimes(r-1)} \simeq \frac{I_{Z}^{(r-1)}}{I_{Z}^{(r-2)} J_{2}}$.

$$
0 \rightarrow \frac{I_{Z}^{(r-1)}}{I_{Z}^{(r-2)} J_{2}} \rightarrow \frac{J_{(r-1)}}{I_{Z}^{(r-2)} J_{2}} \rightarrow \frac{J_{(r-1)}}{J_{2} J_{(r-2)}} \rightarrow \frac{J_{(r-1)}}{J_{r}} \rightarrow 0 .
$$

Since $I_{Z}{ }^{(r-2)} J_{2} \subset J_{2} J_{(r-2)}$ the map $\frac{J_{(r-1)}}{I_{Z}^{(r-2)} J_{2}} \rightarrow \frac{J_{(r-1)}}{J_{2} J_{(r-2)}}$ is surjective. Also $\frac{J_{(r-1)}}{J_{2} J_{(r-2)}} \rightarrow$ $\frac{J_{(r-1)}}{J_{r}} \rightarrow 0$, since $J_{2} J_{(r-2)} \subset J_{r}$. There exists an effective divisor $N_{(r-2)}$ on $Z$ such that

$$
\frac{J_{(r-1)}}{J_{r}} \simeq\left(\frac{I_{Z}}{J_{2}}\right)^{\otimes(r-1)}\left(N_{(r-2)}\right) .
$$

From (6) we deduce $\frac{J_{(r-1)}}{J_{r}} \simeq \wedge^{2}\left(\frac{J_{(r-1)}}{I_{Z} J_{(r-1)}}\right) \otimes M_{(r-1)}{ }^{\otimes-1}$. Thus,

$$
\wedge^{2}\left(\frac{J_{(r-1)}}{I_{Z} J_{(r-1)}}\right) \simeq \frac{J_{(r-1)}}{J_{r}} \otimes M_{(r-1)},
$$

$\wedge^{2}\left(\frac{J_{(r-1)}}{I_{C} J_{(r-1)}}\right)$

$$
\simeq \omega_{Z}{ }^{-(r-1)}(-(N+1)(r-1)) \otimes O_{Z}\left((r-2) m-(r-1) D_{1}+D_{(r-1)}\right) .
$$

Proposition 2. Let $Z$ be an irreducible nonsingular $(N-2)$-subvariety in $\mathbb{P}^{N}, r Z=$ $F \cap X, r \in \mathbb{N}, r \geq 2$, where $F$ is a smooth hypersurface of degree $m, X$ is a normal hypersurface of degree $n, Z \cap \operatorname{Sing}(X) \neq \emptyset$. Then $\frac{J_{(r-1)}}{J_{r}} \simeq \omega_{Z}(-(m+n-(N+1)))$. There are effective divisors $V$ and $D_{1}$ on $Z$ such that $O_{Z}\left(V-(r-1) D_{1}\right) \simeq \omega_{Z}{ }^{r}(-r m-n+$ $(N+1) r)$.

Proof. Since $r Z=F \cap X, J_{r}$ is a locally complete intersection ideal. We see that $\omega_{r} \simeq$ $O_{r Z}(m+n-(N+1))$.

As we have seen in Section 1, letting $D_{1}$ be the associated divisor to the torsion subsheaf $\frac{M_{1}}{O_{Z}(-m)}$, we have $M_{1}=O_{Z}\left(D_{1}-m\right)$. We shall see that $\frac{J_{(r-1)}}{J_{r}} \simeq \omega_{Z}(-(m+n-$ $(N+1)))$.

Let $S=\frac{J_{(r-1)}}{J_{r}}$. The exact sequence

$$
0 \rightarrow S \rightarrow \frac{O_{\mathbb{P}^{N}}}{J_{r}} \rightarrow \frac{O_{\mathbb{P}^{N}}}{J_{(r-1)}} \rightarrow 0
$$

induces the surjective map

$$
\omega_{r Z} \simeq \mathcal{E} x t^{2}\left(O_{r Z}, \omega_{\mathbb{P}^{N}}\right) \rightarrow \mathcal{E} x t^{2}\left(S, \omega_{\mathbb{P}^{N}}\right),
$$

since $O_{(r-1) Z}$ is locally Cohen-Macaulay and hence $\mathcal{E} x t^{3}\left(O_{(r-1) Z}, \omega_{\mathbb{P}^{N}}\right)=0$.

$\mathcal{E} x t^{2}\left(S, \omega_{\mathbb{P}^{N}}\right)$ is a rank 1 locally free sheaf on $Z$.

$$
\begin{aligned}
S & \simeq \mathcal{E} x t^{2}\left(\mathcal{E} x t^{2}\left(S, \omega_{\mathbb{P}^{N}}\right), \omega_{\mathbb{P}^{N}}\right) \simeq \mathcal{E} x t^{2}\left(\left.\left(\omega_{r Z}\right)\right|_{Z}, \omega_{\mathbb{P}^{N}}\right) \\
& \simeq \mathcal{E} x t^{2}\left(O_{Z}, \omega_{\mathbb{P}^{N}}\right)(-l) \simeq \omega_{Z}(-l),
\end{aligned}
$$

where $l=m+n-(N+1)$. 
The functor $\mathcal{E} x t^{2}\left(-, \omega_{\mathbb{P}^{N}}\right)$ is exact and reflexive on the category of Cohen-Macaulay $O_{\mathbb{P}^{N}}$-modules of codimension 2. Thus, $\frac{J_{(r-1)}}{J_{r}} \simeq \omega_{Z}(-(m+n-(N+1)))$.

By Proposition $1 \frac{J_{(r-1)}}{J_{r}} \simeq\left(\frac{I_{Z}}{J_{2}}\right)^{\otimes(r-1)}\left(N_{(r-2)}\right)$, so

$$
\begin{aligned}
\omega_{Z}(-(m+n- & (N+1))) \\
& \simeq \omega_{Z}{ }^{-r+1}((r-1)(m-(N+1))) \otimes \mathcal{O}_{Z}\left(-(r-1) D_{1}\right) \otimes O_{Z}\left(N_{(r-2)}\right) .
\end{aligned}
$$

Let $V:=N_{(r-2)}$. It follows $O_{Z}\left(V-(r-1) D_{1}\right) \simeq \omega_{Z}{ }^{r}(-r m-n+(N+1) r)$.

2.

Definition 3. A closed subscheme $Z \subset \mathbb{P}^{N}$ of codimension $k$ is a complete intersection if there are $k$ hypersurfaces (i.e. locally principal subschemes of codimension 1) $F_{1}$, $F_{2}, \ldots, F_{k}$, such that $Z=F_{1} \cap \ldots \cap F_{k}$, as schemes.

Proposition 4. Let $Z \subset \mathbb{P}^{N}$ be a reduced irreducible subvariety of codimension 2 . Let $Y$ be an irreducible hypersurface in $\mathbb{P}^{N}, Z \subset Y$. Let $\omega^{o}{ }_{Z}$ be the dualizing sheaf of $Z$. Then there exists a hypersurface $X$ in $\mathbb{P}^{N}$ such that $Z=Y \cap X$ is a scheme-theoretical complete intersection if and only if $Z$ satisfies the following properties

- $\left.\omega^{o}{ }_{Z} \simeq \omega_{\mathbb{P}^{N}} \otimes \wedge^{2} \mathcal{N}_{Z}\right|_{\mathbb{P}^{N}}$.

- $\operatorname{deg} Y$ divides $\operatorname{deg} Z$.

- $\omega^{o}{ }_{Z} \simeq O_{Z}\left(\operatorname{deg} Y+\left(\frac{\operatorname{deg} Z}{\operatorname{deg} Y}\right)-N-1\right)$.

Proof. For $N=3$ see [1] and [3]. Let us assume $\operatorname{deg} Y=m$. Let $\left.\omega^{o} Z \simeq \omega_{\mathbb{P}^{N}} \otimes \wedge^{2} \mathcal{N}_{Z}\right|_{\mathbb{P}^{N}}$. Let $\frac{\operatorname{deg} Z}{\operatorname{deg} Y}=n$ and $\omega^{o} Z \simeq O_{Z}(m+n-N-1)$.

Let $\mathcal{N}$ be the normal bundle of $Z$ in $\mathbb{P}^{N}$. Let $\mathcal{E}$ be a rank 2 vector bundle in $\mathbb{P}^{N}$ obtained from $Z$ via Hartshorne-Serre correspondence so that $Z \subset \mathbb{P}^{N}$ is the set of zeroes of a section $\tilde{s} \in H^{0}\left(\mathbb{P}^{N}, \mathcal{E}\right)$ and $\left.\mathcal{E}\right|_{Z} \simeq \mathcal{N}$. Let $\mathcal{E}^{*}$ be the dual of $\mathcal{E}$. Let $I_{Z}$ be the ideal sheaf of $Z$ in $\mathbb{P}^{N}$.

We have an exact sequence

$$
0 \rightarrow \operatorname{det} \mathcal{E}^{*} \rightarrow \mathcal{E}^{*} \rightarrow I_{Z} \rightarrow 0
$$

Since $\left.\omega^{o}{ }_{Z} \simeq \omega_{\mathbb{P}^{N}} \otimes \wedge^{2} \mathcal{N}_{Z}\right|_{\mathbb{P}^{N}}$

$$
\omega_{Z}^{o} \simeq \wedge^{2}\left(\frac{I_{Z}}{I_{Z}^{2}}\right)^{*}(-N-1) .
$$

Since $\omega_{Z}^{o} \simeq O_{Z}(m+n-N-1)$,

$$
\begin{gathered}
\wedge^{2}\left(\frac{I_{Z}}{I_{Z}^{2}}\right)^{*} \simeq O_{Z}(m+n), \\
\left.\wedge^{2} \mathcal{E}^{*}\right|_{Z} \simeq \wedge^{2}\left(\frac{I_{Z}}{I_{Z}^{2}}\right) \simeq O_{Z}(-m-n), \\
\wedge^{2} \mathcal{E}^{*} \simeq O_{\mathbb{P}^{N}}(-m-n) .
\end{gathered}
$$

The first Chern class of $\mathcal{E}^{*}$ is $-m-n$, the second Chern class is $m n$.

From (7) we have

$$
0 \rightarrow O_{\mathbb{P}^{N}}(-m-n) \rightarrow \mathcal{E}^{*} \rightarrow I_{Z} \rightarrow 0 .
$$


Tensoring with $-\otimes O_{\mathbb{P}^{N}}(m)$, we obtain

$$
0 \rightarrow O_{\mathbb{P}^{N}}(-n) \rightarrow \mathcal{E}^{*}(m) \rightarrow I_{Z}(m) \rightarrow 0 .
$$

Computing cohomology,

$$
H^{0}\left(\mathcal{E}^{*}(m)\right) \rightarrow H^{0}\left(I_{Z}(m)\right) \rightarrow 0,
$$

since $H^{1}\left(O_{\mathbb{P}^{N}}(-n)\right)=0$. Let $s_{1} \in H^{0}\left(\mathcal{E}^{*}(m)\right)$ be an element whose image is equal to $Y$ in $H^{0}\left(I_{Z}(m)\right)$. For every effective divisor $D$ in $\mathbb{P}^{N}$, there exists a commutative diagram

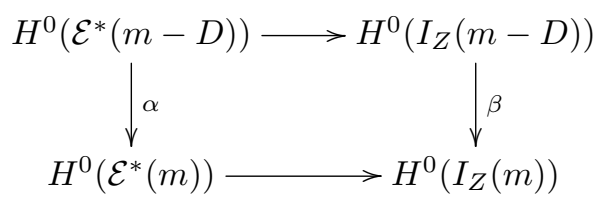

with vertical arrows $\alpha: H^{0}\left(\mathcal{E}^{*}(m-D)\right) \rightarrow H^{0}\left(\mathcal{E}^{*}(m)\right)$ and $\beta: H^{0}\left(I_{Z}(m-D)\right) \rightarrow$ $H^{0}\left(I_{Z}(m)\right)$ for $s_{1} \in H^{0}\left(\mathcal{E}^{*}(m)\right)$ is not in $\operatorname{Im} \alpha$ since $Y$ is irreducible. Thus, the scheme of zeroes of $s_{1}$ is empty in codimension 1 components.

$c_{2}\left(\mathcal{E}^{*}(m)\right)=c_{2}\left(\mathcal{E}^{*}\right)+m c_{1}\left(\mathcal{E}^{*}\right)+m^{2}=0$, so the scheme of zeroes of $s_{1}$ is empty. Note that $c_{1}\left(\mathcal{E}^{*}(m)\right)=c_{1}\left(\mathcal{E}^{*}\right)+2 m=m-n$. Thus, $\mathcal{E}^{*}(m)$ is decomposable into the direct summands $O_{\mathbb{P}^{N}}$ and $O_{\mathbb{P}^{N}}(m-n)$.

Hence, we deduce that $\mathcal{E}^{*}$ is decomposable into the direct summands $O_{\mathbb{P}^{N}}(-m)$ and $O_{\mathbb{P}^{N}}(-n)$. Therefore, there exists a hypersurface $X$ of degree $n$ such that $Z=Y \cap X$.

The necessity condition is obvious since $Z=Y \cap X$ is a scheme-theoretical complete intersection.

\section{References}

[1] M. Boratyński, On the curves of contact on surfaces in a projective space, in: Algebraic K-Theory, Commutative Algebra, and Algebraic Geometry, Contemp. Math. 126, Amer. Math. Soc., Providence, RI, 1992, 1-8.

[2] M. R. Gonzalez-Dorrego, Degree 8 and genus 5 curves in $\mathbb{P}^{3}$ and the Horrocks-Mumford bundle, in: Algebraic Geometry and Singularities, Progr. Math. 134, Birkhäuser, Basel, 1996, 303-310.

[3] S. Greco, Alcune osservazioni sui sottoschemi di codimensione 1 di una varieta proiettiva, Univ. Bologna, Seminari di Geometria 1985, 89-100.

[4] P. Griffiths, J. Harris, On the Noether-Lefschetz Theorem and some remarks on codimensiontwo cycles, Math. Ann. 271 (1985), 31-51.

[5] S. Katz, Genus zero Gopakumar-Vafa invariants of contractible curves, J. Differential Geom. 79 (2008), 185-195.

[6] R. Pandharipande, A. Zinger, Enumerative geometry of Calabi-Yau 5-folds, in: New Developments in Algebraic Geometry, Integrable Systems and Mirror Symmetry, Adv. Stud. Pure Math. 59, Math. Soc. Japan, Tokyo, 2010, 239-288. 
